\title{
La Política de Financiamiento de la Educación Básica en Chile (1980 -2008): Consecuencias y Disyuntivas
}

\author{
The Policy of Financing Elementary Education in Chile \\ (1980-2008): Consequences and Disjunctive
}

Sebastián Donoso Díaz'

Resumen:

El artículo analiza los elementos céntrales de la política de financiamiento de la educación básica chilena impulsados desde al reforma neoliberal del año 1980 que cambió el sistema de financiamiento por uno de subsidio a la demanda. El texto revisa la situación del sistema, los principales resultados después de casi tres décadas de aplicación y la búsqueda de solución a los problemas de incremento de segmentación social y de la reproducción intergeneracional de las diferencias sociales, atribuibles por algunos a los fallos de mercado y por otros fundamentalmente a problemas técnicos de fijación del valor del subsidio. El trabajo analiza las proyecciones del problema, que pese a las enmiendas, se cree no logra fusionar dos lógicas irreductibles que subyacen: la de Estado Subsidiario vigente actualmente, fuerte y dominante y las de Estado Solidario que no logra avanzar. Este debate estará presente en un horizonte próximo e implicará un punto de inflexión importante para decidir cómo solucionar los problemas que ha generado.

Palabras clave: Desigualdades educacionais; Estados Unitários; Estados Federais; Políticas

educacionais; Financiamento da educação.

\footnotetext{
${ }^{1}$ Doctor en Educación, Académico del Instituto de Investigación y Desarrollo Educacional de la Universidad de Talca, Chile. sdonoso@ utalca.cl. Este trabajo es parte del Proyecto PSD - 60, financiado por el fondo CONICYT - PBCT, Chile.
} 
Abstract:

The article analyzes the central elements of the political funding of basic education Chilean driven from the neo-liberal reform of 1980 that changed the system of funding by one of subsidy demand. The text reviews the status of the system, the main results after nearly three decades of implementation and finding solutions to the problems of increasing social segmentation and the intergenerational reproduction of social differences, attributed by some to market failures and otherwise mainly to technical problems of fixing the value of the grant. The paper analyzes projections of the problem, that despite the amendments, it believes fails to merge two irreducible underlying logic: the State of Subsidiary currently in force, strong and dominant and the State of solidarity that fails to make progress. This debate will be present in a near horizon and comprise an important turning point in deciding how to solve the problems it has generated.

Keywords: Funding for education; basic education; education allowance; State Subsidiary; New design of State. 


\section{Presentación.}

Elartículoanalizalaevoluciónquehaseguidola política de financiamiento de la educación básica (primaria) en Chile prácticamente en las últimas tres décadas, en razón que ha experimentado cambios relevantes en su orientación y magnitud. La exposición se estructura en referencia a cuatro temáticas centrales: Primero, una breve síntesis de los principales cambios experimentados en el sistema escolar básico en los años en estudio. Segundo, análisis de los principios y mecanismos de aplicación de la actual política de financiamiento de la educación básica en Chile, la que fue instalada a comienzos de la década de los años 80 y que con adecuaciones ha seguido operando a la fecha bajo el mismo racional que fue ideada. Tercero, identificación de algunas consecuencias derivadas de esta política y las eventuales correcciones de corto plazo diseñadas dentro del paradigma dominante; para finalmente, en cuarto lugar, exponer ciertas disyuntivas de la política de financiamiento en el escenario más probable que se avecina para la educación chilena.

Algunos considerando que sustenta la visión asumida en el trabajo dicen relación, en primer lugar, conelhechoquelapolítica definanciamiento de la educación es constitutiva sino tributaria de la política educacional, por ende, analizarles como fenómenos desvinculados implica una visión tecnocráctica que asume que los principios y mecanismos de financiamiento son neutrales, algo que se contradice en si misma, ya que una política busca dar orientaciones y señales para direccionar los resultados que se busca alcanzar. Consistente con ello, una política en este ámbito se orienta a determinados objetivos, en razón de lo cual es indispensable establecer sus directrices para evaluar su pertinencia y luego, el grado de éxito alcanzado. Finalmente, la política de financiamiento de la educación básica o primaria, como parte de la política general de financiamiento de la educación, responde en su funcionalidad al todo, aunque posee objetivos particulares que la identifican.

\section{Breve síntesis de los cambios experimentados entre los años 1980 al 2008}

\subsection{Caracterización del Sector de Educación Básica chileno.}

En Chile, la gratuidad de la enseñanza primaria es uno de los pocos principios que desde inicios del siglo XX no ha sido puesto en duda. Desde su proclamación por el Estado en el año 1860, pasando por las leyes de instrucción primaria obligatoria de 1920 y 1925, la reforma de 1927 y los diversos intentos de masificación de la educación en la década del 40, hasta la reforma educacional de 1965 (que elevó de seis a ocho grados la educación básica), es una de los extraños casos consensuados de política pública en el país, sustentado en la contribución de la educación en la promoción del crecimiento y la equidad en la sociedad y a su asimilación como un bien público.

Consistente con lo señalado, el gasto público es y ha sido la principal fuente de financiamiento para la educación de la mayoría de la población, y pese a la incorporación del sector privado, se mantiene como la fuente más relevante de la enseñanza primaria (OCDE, 2005).

Pese a la deseabilidad de las políticas impulsadas en el país por fortalecer la educación primaria, Chile tardó más de 60 años en universalizar la educación básica para toda la población en edad escolar, y a la fecha (2008) aún resta una importante brecha de cobertura para universalizar la enseñanza secundaria (8\%), proclamada obligatoria por el Estado a partir del año 2003.

Al año 2006 el sistema escolar chileno, desde el nivel preescolar al secundario, está conformado por algo más de 3,6 millones de estudiantes. Cifra que se encuentra estabilizada desde el año 2002 con variaciones menores que no superan los 70 mil alumnos sobre ese valor. Para la enseñanza primaria esta situación es algo diferente. El año 2001 representó su cifra mayor con 2,36 millones de alumnos, descendiendo a 2,14 millones en el 2007; de igual forma la representación de sus estudiantes en el total del sistema en esos años 
se redujo del 66 al 60\% (MINEDUC, 2007). El $87 \%$ de la matrícula es urbana, tendencia que ha aumentado irreversiblemente año en año.

\subsection{Principales cambios en las Políticas de Financiamiento de la Educación.}

La fractura de la democracia chilena del año 1973 implicó el rediseño de la sociedad en sus diversos ámbitos. El sector educación fue una de las áreas que más tardíamente se ajustó al modelo de economía de mercado de inspiración neoliberal impulsado en Chile desde el año 1976, ello ocurrió por el hecho que en el seno de la misma Dictadura cívico/militar existían indecisiones y disputas entre dos corrientes de acción diferentes: una ligada a la visión más tradicional del Estado en este campo, con un fuerte rol del sector público en la provisión de educación, y otra que propugnaba decididamente su apertura a la privatización, enfatizando entre otros aspectos la incorporación de proveedores privados de educación. Este debate se zanjó finalmente a mediados del año 80 (Directivas Presidenciales para la Educación, 1980), imponiéndose la perspectiva privatizadora, lo que derivó en cambios de gran relevancia en las políticas e instrumentos de financiamiento de la educación pública en todos sus niveles.

De esta forma el año 1980 es el último en el cual el Estado chileno financió con recursos públicos la oferta y la provisión directa de educación. Hasta esa fecha, más del $85 \%$ del total de la matrícula del sistema escolar asistía a establecimientos estatales cuyo financiamiento correspondía a la provisión total de los recursos por parte del Estado (oferta), sin que existiesen mecanismos relevantes asociados a la demanda efectiva de alumnos que cada establecimiento atendía.

La reforma al sistema de financiamiento aplicada a partir del año 1981 fue una de las transformaciones más eficaces impulsadas en el sector educación en los últimos 40 años, por cuanto no solamente incidió en los criterios, orientaciones y mecanismos de financiamiento, sino que también tuvo y tiene aún impactos relevantes en lo pedagógico, de forma que se trató, finalmente, de una reforma educacional impulsada desde las política de financiamiento del sector ${ }^{2}$.

La nueva política de financiamiento público asume la demanda por educación como el criterio central en la asignación de los recursos. Se aplicó masivamente a partir del año $1981^{3}$, significando cambios en la arquitectura del sistema en tres aspectos claves y ciertamente, en un escenario de fuertes restricciones democráticas que impedía cualquier expresión opositora a estas propuestas, sino a un riesgo muy alto para las personas, en caso contrario habrían resultado muy difíciles de aplicar y menos con esos plazos. Los aspectos claves son: primero, los establecimientos escolares - que en su totalidad pertenecían al Estado nacional - fueron transferidos a los gobiernos locales (municipios). El segundo, es que de igual forma los docentes y personal no docente fueron transferidos a los gobiernos locales, perdiendo un conjunto relevante de beneficios económicos y de carrera profesional que incluso a la fecha aún no recuperan del todo, pese a los reconocidos esfuerzos desplegados por los gobiernos democráticos (ROJAS, 1998). El tercer componente clave es que se estimuló y facilitó la libre entrada/salida de proveedores privados de educación, mediante los nuevos instrumentos financieros de operación.

El nuevo sistema de financiamiento opera desde entonces con un subsidio que se entrega al propietario del establecimiento escolar, sea este el municipio o un empresario privado, el que es calculado a partir del promedio asistencia diaria/estudiante mes que se traduce

2Se enfatiza esta condición pues al revisarse las reformas políticas de la educación chilena, no siempre es consignada como tal. Sin embargo, precisamente su existencia inadvertida como "reforma expresa" es la que facilitó su expansión a todas las áreas del proceso educativo.

${ }^{3} \mathrm{Si}$ bien el proceso se completa el año 1987, más de la mitad de los establecimientos escolares son traspasados ese año a este nuevo sistema de financiamiento, y de paso todo el sistema universitario público también a una nueva modalidad. 
a un valor a recibir. Si bien el sistema tiene otros instrumentos de financiamiento, el subsidio por estudiante sigue siendo el mecanismo central.

\section{La Política de Financiamiento de la Educación Básica vigente desde el año 1981}

\subsection{Los objetivos políticos del nuevo modelo de financiamiento.}

La necesidad de sincronizar el funcionamiento del sector educación con la economía de mercado fue el principio político clave que impulsó el cambio del modelo de financiamiento del sistema educacional. Como tal, este proceso sustitutivo no respondiónecesariamenteauna discusión pública, ni a un diagnóstico que diera cuenta del fracaso del modelo de subsidio a la oferta. La literatura de la época muestra la carencia de estudios empíricos que fundamenten la decisión adoptada (JOFRÉ, 1988). Sus objetivos políticos más evidentes eran dos: consolidar el modelo privado de desarrollo económico, mediante la imposición de un sistema de subsidio basado en el racional privado, tanto para operadores públicos (municipales) como privados, y favorecer la descentralización de la gestión del Estado, traspasando a los gobiernos subnacionales atribuciones en este plano y a paralelamente reduciendo su impacto al fortalecer el accionar de los privados.

Además, la nueva arquitectura implantada buscaba fortalecer los objetivos propuestos mediante un mayor control del gasto público, derivado del sistema de asignación de los subsidios y también, mejorar el rendimiento de los estudiantes mediante el desarrollo y fortalecimiento de la competencia entre los establecimientos públicos y privados, pues supone que por esta vía aumentará la eficiencia, derivando en mejores resultados educacionales.

La complejidad de las tareas que se impulsan con los cambios de 1981 es alta, la que se eleva ante el hecho que las autoridades del gobierno implantan la nueva política de financiamiento de la educación con una fuerte reducción del presupuesto público del sector que alcanzó al 24\% real entre los años 1982 y 1989 (GONZÁLEZ, 2003, p. 610; SAPELLI, 2002, p. 285). Esta situación es crucial para comprender parte del deterioro de los resultados educacionales del país, incluso con impactos que persisten a la fecha, los que suelen subestimarse por los autores proclives a este enfoque, olvidando entre otros aspectos que el nuevo sistema de subsidio creó, entre otros motivos, severos problemas de financiamiento del sector municipal, al extremo que en 1986 tuvo que reajustarse el valor del subsidio en forma relevante para evitar su colapso del sistema (JOFRÉ, 1988).

\subsection{Los instrumentos de financiamiento 4}

Como fue señalado, el subsidio opera como criterio central para destinar los recursos a los establecimientos empleando para ello "el promedio de asistencia diaria a clases del estudiante" por trimestre/móvil (con un tope de 20 días mes). Esta resultante se asocia al valor mes del subsidio, dando la cifra resultante por estudiante, la cual es entregada por el Ministerio de Educación a los empresarios (públicos y privados). Este sistema de subsidio aplicado en Chile, denominado "vales o vouchers lump sum", ha mostrado ser menos eficiente que otros mecanismo equivalentes (HOXBY, 2002).

En Chile, la educación primaria asigna por esta vía la mayor parte de los recursos a los empresarios públicos y privados. El subsidio de cada estudiante de educación básica regular (2008) tiene un precio base mes de US \$75. En el caso de los Municipios, éstos no pueden cobrar valores adicionales a los padres (salvo cifras menores que no suman los US \$ 10 al año por estudiante), no así los empresarios privados, que cumpliendo determinados procedimientos optan al sistema de "financiamiento compartido", que

${ }^{4}$ Se usa como base comparativa el año 2003 dado que se dispone de la mayor cantidad de datos para ello. ${ }^{5}$ US $\$ 1=\$ 480$ pesos chileno (mayo 2008). 
les permite incrementar sus ingresos en valores van entre los US \$ 5 a 15 por alumno mes 5 .

Porsuparte,elMinisterioentregaalosmunicipios recursos para manutención de infraestructura escolar, que suelen ser cifras menores. También el Ministerio interviene en algunos establecimientos públicos con programas directos (destinados a escuelas prioritarias o población focalizada -desertores, etc.) que implican asignación de recursos directos fundamentalmente en textos, materiales, horas de trabajo y asistencia técnica, más que recursos financieros frescos para el municipio. Este proceder se podría catalogar que opera con el racional de subsidio a la oferta y muestra que existen aún algunos instrumentos que confieren al sistema chileno aplicación de instrumentos mixtos, aunque al revisarse las cifras que están involucradas no cabe duda alguna que ello no es así, más del $62 \%$ de los gastos del Gobierno central son referidos a subvenciones y menos del $7 \%$ se asigna en programas directos (DIPRES, 2003).

El 92\% de todas las transferencias que realiza el Ministerio al sector en estos ítems son referidas a la escolaridad (asistencia de alumnos), el restante porcentaje se asigna por compensación de ruralidad, desempeño difícil, internado y otra serie de ponderadores que son secundarios o terciarios respecto de los montos involucrados (DIPRES, 2004). Deigual forma, hasta lo queva del año 2008 inclusive (mayo), existe prácticamente un valor de subsidio para la enseñanza primaria ya que el $93 \%$ del componente es fijo y el $7 \%$ es variable. Este punto es uno de los temas más complejos del debate, y si bien se supone que a partir del año 2008 se aplica un subsidio adicional (denominado subsidio preferencial, aplicado ala población escolar que vive en condiciones de pobreza) el reglamento de operación de este proceso no está aún en operación, por ende no está aún funcionando en régimen.

Una fuente de recursos destinados fundamentalmente a infraestructura y en menor medida a equipamiento proviene de fondos regionales(FondoNacionaldeDesarrolloRegional), que son recursos aportados por el gobierno central para que sean administrados por los gobiernos subnacionales. Su monto al año 2003 destinado a educación no superó el $6 \%$ del asignado a subvención. También cifras mucho menores los municipios destinan recursos financieros para gastos corrientes, fundamentalmente salarios y otros que en cifras totales no superan el 2,5\% de los recursos destinados a subvención, pero que en algunos casos de municipios pequeños esta cifra es relevante.

Finalmente, en el caso chileno el aporte privado es de significación. Del 7,3\% del PIB aportado a educación (2006), el 46\% es de fondos privados, situación que se explica fundamentalmente por la educación superior, sin embargo la participación del gastos privado en todos los niveles es un fenómeno cierto que requiere un análisis más detallado, el que se realiza en la sección siguiente.

\section{Resultados y Correcciones de la Política de Financiamiento de la Educación Básica.}

\subsection{Análisis del modelo de subsidio y sus condicionantes}

En su aplicación en Chile el sistema de financiamiento mediante subsidio ha tenido y a su vez ha generado muchos problemas. Algunos provienen de la forma cómo fue implantado, otros de su diseño y otros de los supuestos y considerandos que asume. Dependiendo de la naturaleza del problema, en el primer grupo se sitúan prioritariamente aquellos críticos que difieren radicalmente con la concepción política del tema, en la segundo perspectiva se encuentran los que comparte en modelo pero tienen ciertos reparos técnicos, mientras que en el tercer grupo se identifica a los que tiene reparos técnicos porque no comparten el modelo.

Las críticas a la legitimidad del modelo y su implantación se refieren tanto al origen viciado de su diseño y aplicación, ocurrido en pleno período dictatorial y sin posibilidades de participación social. Concomitantemente, aluden al afán privatizador de todas las medidas propuestas, incluyendo la descentralización 
de la gestión del Ministerio de Educación y el traspaso de establecimientos y docentes a dependencias de los gobiernos locales.

El segundo grupo, a saber, quienes tienen diferencias en materia técnica comparten los fundamentos y principios operativos del subsidio y sus mecanismos de aplicación. Los puntos en desacuerdo residen básicamente en que establecer un sistema de subsidio de precio único con pago similar para todos (como es el caso chileno), subsidia más a quien tiene mayor capital humano inicial, es decir genera un efecto perverso que favorece a los que tienen costos formativos menores, perjudicando por esta vía a los más pobres (SAPELLI, 2002, p. 287).

Este es quizás uno de los principales problemas de corto plazo que generó la aplicación de esta política de financiamiento, lo que derivó finalmente en un problema de mediano y largo plazo socialmente difícil de solucionar: el reforzamiento de un sistema educacional altamente segmentado en lo social y educacional, con importantes diferenciales de calidad. Cuesta entender prácticamente se haya tardado 27 años (comienza a regir el 2008) en generar un valor de subsidio diferente para un segmento de la población escolar más pobre, ya que había sido denunciado por autores de todas las corrientes de opinión como un tema capital que debía repararse. La actual ley de subsidio preferencial, otorga un 30\% adicional de recursos por estudiante (US \$ 38) perteneciente al segmento más pobre, además de asignar ingresos complementarios a los establecimientos que tienen al menos el 15\% de población perteneciente a los segmentos sociales más pobres. De esta forma el sistema hoy debería tener dos niveles el valor regular y el preferencial. Según los partidarios del modelo el tema "precio" era finalmente el que desvirtuaba el sistema de subsidio y fue el que generó la estratificación señalada. Es decir, con precios ajustados al costo de la formación por nivel socioeconómico ese problema no debió haberse generado. Para los más críticos se trató de una consecuencia inevitable del sistema de mercado y que los subsidios diferenciados corrigen sólo parcialmente esta situación.

En otra materia, los partidarios del modelo sostienen que la existencia de subsidios a la oferta, esto es, algunos programas que aportan recursos preferentemente a los establecimientos municipales, se transforma en un impuesto "al traslado de los estudiantes a los establecimientos privados", pues castiga a quienes se cambian del sector municipal al privado. Los hechos han demostrado que este fenómeno no sigue "la crítica señalada", por el contrario, la revisión de la evolución de la matrícula de estudiantes por tipo de establecimiento escolar (municipal / particular subvencionado) da cuenta de cambios de magnitud ocurridos entre los años 1980 y 2007. En síntesis, el sector municipal tenía el año 1980 el $80 \%$ de matrícula de niños y pasa a tener el $50 \%$ el 2007, proceso que ha sido gradual y permanente, compartiendo la matrícula actualmente en igual proporción ambos sectores. Para el caso específico de la enseñanza básica pública ${ }^{6}$, la situación cambia en parte, ya que el sector municipal agrupa al 53\% de la matrícula, ante el 47\% del subvencionado (MINEDUC, 2008).

El tema expuesto evidencia algunos de los problemas derivados de la implantación del modelo. Como fue señalado, la segmentación del sector educación en varios cuasimercados (ALMONACID y otros, 2008) que se transforman en segmentos con escasa interacción en vez de mercados dinámicos. Una muestra de lo señalado es que la educación rural, que en el caso chileno concentra a los grupos sociales de menores ingresos es atendida en un $85 \%$ por los establecimientos municipales, teniendo los privados una presencia exigua. Este modelo de financiamiento produce un sistema de señales

${ }^{6}$ Se asume por educación pública aquella que es financiada en grado relevante por el Estado, en el caso chileno considera tanto los establecimientos de dependencia pública municipal, como los particulares que se acogen a esta normativa. 
que no es funcional a la realidad rural. El supuesto esencial que sustenta el modelo, a saber: "el financiamiento sigue a la calidad", no es válido universalmente y requiere de un sinnúmero de condiciones que no se cumple, aún tras las tres décadas los de aplicación. El modelo presume que a mayor asistencia de los estudiantes a clases se genera más aprendizaje y por ende mayor calidad. Sin embargo su aplicación omite una serie de considerandos claves para comprender los resultados alcanzados en Chile en este período de tiempo que distan de lo "esperado": (i) las diferencias de capital humano inicial de la población escolar; (ii) los recursos asociados a la producción de una educación de calidad según las diversas dotaciones de capital; (iii) acceso y capacidad de comprensión de la información necesaria para adoptar las decisiones de parte de las familias (simetría de información); (iv) oferta territorial uniforme de educación de calidad; y (v) mecanismos técnicos adecuados para implantar y controlar el sistema de asignación de recursos (DONOSO y SCHMAL, 2008).

\subsection{La segmentación confirmada: análisis del gasto en educación por estrato de ingresos de las familias}

La complejidad que reviste la segmentación se registra de manera más explícita al revisar los antecedentes delgastoeneducaciónquerealizan las familias según el estrato socioeconómico al que pertenecen. La segmentación social es un tema sustantivo del diseño e implementación de las políticas públicas, la respecto, si bien no es exigible al sistema educativo que por sí mismo resuelva las profundas desigualdades presentes, es legítimo reclamarle que sea capaz de reducirlas significativamente en cada generación. La velocidad de este proceso se entiende directamente relacionado con la calidad de los servicios educacionales que la sociedad es capaz de proporcionar, esta materia es la que está en duda y reclama una revisión de la estrategia que como país se ha implementado en el sector educación, pues las diferencia iniciales no han podido - en los hechos - reducirse.
Las cifras de gastos confirman que en promedio se gasta en un alumno del quintil más rico casi el triple que en uno del quintil más bajo. Esta brecha está presente en todos los niveles educativos. El gasto por alumno del quintil de mayores ingresos es 16 veces superior al de menores recursos, distancia que se compensa parcialmente debido a la focalización del gasto público (DONOSO y HAWES, 2002, p.36; MARCEL y TOKMAN, 2005, p. 43).

En razón de lo expuesto no debiera extrañar que los resultados en logros de aprendizaje medidos en las pruebas nacionales (SIMCE) del sistema educacional chileno no suban y permanezcan estancados por casi una década - de 1999 a 2007- (MINEDUC, 2008), pues se entiende que el costo de educar ala población más pobre es muy superior y el sistema de subsidio vigente hasta el año 2007 no consideraba ese componente, y pese a que el gasto público por alumno del quintil más pobre duplica al del quintil más rico, es claramente insuficiente para poder compensar tales diferencias.

En teoría el sistema vigente en Chile garantiza a toda la población escolar el acceso a una educación de calidad, aún cuando las familias no dispongan de los recursos financieros. Esto se logra merced al financiamiento público que complementa los aportes que las familias estén dispuestas a incurrir (financiamiento privado). Por otra parte, en teoría también el sistema imperante garantiza la libre elección del establecimiento por las familias, posibilitando el desarrollo de un mercado competitivo que induciría a incrementar la calidad de la educación y la equidad en la población. Sin embargo los hecho contradicen el fondo de estas concepciones, no se ve razón alguna para que los resultados de la educación pública fueran superiores, y la evidencia es suficientemente fuerte para sustentar que el mercado no ha regulado por la vía de la demanda los principales fallos.

4.3. Eficiencia interna y externa del sistema educativo: Evidencias y debate

Como fue señalado anteriormente, en los 28 años de implantación del sistema de financiamiento 
por subsidio a la demanda se ha producido en Chile un traspaso de parte de los estudiantes de los establecimientos municipales hacia particular subvencionado. Para algunos esto es fruto de la mayor calidad de los establecimientos particulares, para otros es parte de la segmentación social que deriva en que los menos pobres van abandonando el sistema de educación municipal.

Los resultados de eficiencia interna del sistema educativo registrados a partir del año 1995 y hasta el 2006 (en que hay cifras comparativas) se desplazaron positivamente. La cobertura de la educación básica subió del 92 al 99\%, la tasa de deserción en básica se redujo del 4 al 1.7\%, de igual forma, la tasa de alumnos aprobados en enseñanza básica subió del 92,3a 96\% (MINEDUC: 2007). Sin embargo, el impacto de los resultados reseñados sobre los aprendizajes de los alumnos en el sistema escolar no tiene la progresión esperada respecto de los insumos incorporados, incluyendo los fuertes aportes financieros públicos. Una cifra es elocuente, desde el año 1999 hasta el 2007 (MINEDUC, 2008) incluido, los rendimientos medidos en las Pruebas SIMCE son equivalentes o similares lo que implica que están estancados:

La evidencia aportada por la comparación de los resultados SIMCE a lo largo de los 90 puede ser resumida en cuatro constataciones. Primero, que hay una tendencia consistente aunque leve, de incremento en los promedios nacionales en la primera mitad de la década, reduciendo así levemente las diferencias de logros entre establecimientos municipales y particulares pagados. Esta se estancó de 1996 a 2002 y la diferencia aumentó. Segundo, que la distribución social de los aprendizajes exhibe una distribución altamente estratificada e inequitativa, similar a la de 1990. Tercero, que las mejoras en rendimiento son mayores que las del promedio en el caso de las escuelas básicas que han sido objeto de programas focalizados como el P-900 o el Programa rural, Io que ha significado disminución de las brechas de rendimiento entre este alumnado -el más pobre- y el resto del país. Por último, que las diferencias de logros en el aprendizaje entre las distintas dependencias del sistema subvencionado (Municipal y Particular) son mínimas y no siempre favorables a la educación privada, cuando se comparan grupos socioeconómicos homogéneos (OCDE, 2004, p. 39-40).

El informe de la OCDE permite formarse un panorama cierto del problema. La educación chilena avanzó lentamente hasta finalmente estancarse, siendo más relevante que el factor dependencia del establecimiento (si es público o privado) el factor socioeconómico de la familia, lo que confirma que la sociedad chilena es la segunda más desigual de América Latina tras Brasil, y es una de las más desiguales del mundo (PNUD, 2003). En consecuencia el principal factor explicativo en los resultados escolares son los antecedentes socioeconómicos, variable que ha sido y sigue siendo dominante al extremo que relega otras - como dependencia - con aportes marginales (DONOSO y HAWES, 2002). Adicionalmente esta relación evidencia que la educación tiene un papel limitado en la reducción de la equidad y desigualdad. Lo que refuerza ante el hecho que la detención de los resultados responde también al aumento de la brecha de inequidad en la distribución del ingreso en Chile, que aunque ha implicado reducción de la pobreza las brechas con los de mayores ingresos se incrementan (MIDEPLAN - MINEDUC, 2004).

Los resultados escolares de las pruebas internacionales TIMMS (2003) y PISA del 2006, muestran a Chile muy relegado ante los países más desarrollados, ratificando con ello dos hipótesis. La primera que los logros de Chile son insatisfactorios en toda estructura comparativa, es decir también los establecimientos escolares de elite social y económica del país no alcanzan logros comparables a los de sus pares de otras latitudes. Ello implica que el problema en Chile tiene una dimensión estructural importante y, que los establecimientos particulares, comparados con sus pares están significativamente más atrás. De forma que privatizar la educación no es un camino a seguir, más aún si sus precios promedio (valor colegiatura) triplican el valor de la subvención básica, no siendo sus resultados acordes a este diferencial de inversión. La segunda hipótesis es que en las pruebas internacionales los países con mayor desigualdad social reproducen en parte importante su mayor desigualdad educativa. 
El 'aceptado' estancamiento de los resultados enmateria deaprendizaje sehatransformadoenel eje de un problema mayor cuya resolución podría precipitar una nueva reforma educativa, proceso que podría ser visto como contrarreforma, según se le defina. Por un lado los resultados actuales son explicados sólidamente por la lógica de la causalidad estructural, la cual posterga toda opción de mejoría substantiva de los logros educacionales a un cuadro muy diferente del actual en materia de relaciones sociales del país. Por otro lado, quienes comparten los postulados del sistema de vales o vouchers sostiene que se trata de un tema de eficiencia y de gestión del uso de los recursos más que de diferencias sociales irremontables para el sistema educacional. Sin embargo la explicación tiene otros acentos, algunos autores - Aedo (1988); Aedo y Larrañaga (1995) - señalaban que los establecimientos particulares con subsidio tenían mejores resultados que los establecimientos municipales. Posteriormente Mizala y Romaguera (2003, 2004), controlando los resultados por el capital humano inicial, indican que no habrían diferencias significativas en los resultados; a esta línea también se suma Tokman A. (2001). Un grupo de autores, Sapelli y Vial (2002), Gallego (2002), encuentran que hay diferencias y que debidamente controladas por capital humano son favorables a los establecimientos privados subvencionados. Por otra parte Donoso y Hawes (2002), señalan que el factor clave es el capital social y económico familiar, y Hsieh y Urquiola (2002), amplían esta explicación señalando que los resultados se producen por el traslado de los menos pobres a los establecimientos particulares con subvención del Estado

Adicionalmente, esimportantecomprenderque el mejoramiento de los indicadores de eficiencia interna no implica necesariamente un incremento de la calidad de los aprendizajes, como de hecho se puede deducir de los registros de las pruebas nacionales e internacionales para la presente década. Ello ha dado origen a un nuevo proceso de exclusión social, que cambia los parámetros anteriores establecidos del acceso o no acceso a la educación o del haber completado o no determinados ciclos o niveles. El nuevo proceso de exclusión implica logros formales equivalentes pero diferenciales de calidad muy desiguales, esto se traduce en que existen segmentos de mala calidad de la educación, asociados también precarias condiciones de vida, que generan un círculo de mala calidad.

Estas nuevas formas de exclusión social reproducen la desigualdad intergeneracional sólo que el punto inicial de educación formal es más alto, pero los resultados son los mismos. Los estudiantes que se inician en los círculos de mala calidad de educación primaria, lo continúan en la educación secundaria y finalmente, muchos de ellos acceden a la educación superior de mala calidad. Por ende, no logran dar el salto generacional que la educación promete en su lectura más optimista del Capital Humano. Lo inverso también se confirma, los resultados educacionales corroboran que los con mejor dotación inicial de capital social y cultural alcanzan mejores resultados y por esta vía se mantienen las diferencias sociales, reproduciéndose y perpetuándose la desigualdad, sólo que con nuevos rostros y nuevas dinámicas, pero con un credencialismo formal superior aunque en los hechos estas "credenciales" tienen menos valor.

\section{Disyuntivas para una política sustentable de financiamiento de la educación chilena}

El análisis final parte de la premisa que los problemas de corto plazo serán resueltos tanto por la nueva Ley de Subvención Preferencial, como también por las incrementos basales de los valores de los diversos tipos de subsidio. En segunda instancia, la política de financiamiento es de carácter macro, de forma que considera a todos los niveles del sistema, aunque debe tener acentos propios para cada uno.

Por lo tanto las disyuntivas respecto de la sustentabilidad de una política de financiamiento de la educación pública chilena, dicen relación con cómo abordar los problemas de mediano 
y largo plazo que se generan como también que se arrastran históricamente producto de lo obrado. Un considerando esencial a tener en cuenta en este proceso, es que el sustrato social sobre el cual funciona el sistema educacional es una sociedad altamente desigual, tratándose de una desigualdad severa que conlleva una profunda injusticia (RAWLS, 1986). Por lo cual las respuestas que se generan se insertan en un escenario con cierto nivel de inestabilidad sino impaciencia de los actores más postergados.

El problema estratégico clave del sistema es su alta segmentación social y económica que se traduce en diferenciales de calidad que no alcanzan a ser corregidos por la escuela, ratificándose y reproduciéndose las diferencias intergeneracionales.

El sistema educacional y fundamentalmente el sistema de financiamiento debería entrar en crisis ${ }^{7}$ en un plazo próximo. Este proceso no estará referido un factor puntual sino al diseño político del sistema educacional en razón que algunas de sus funciones políticas claves en materia de fomento de la cohesión y de legitimación del orden social se encuentran severamente comprometidas debido a los grandes problemas que enfrenta la sociedad chilena evidenciados: fuerte reproducción de la desigualdad intergeneracional e incubación de nuevas formas de exclusión social que operan con formatos diferentes a los usualmente conocidos.

Esta crisis será de carácter estratégico, entre otros motivos porque durante casi dos décadas los gobiernos democráticos han buscado compatibilizar dos racionales asentados en fundamentos y criterios de operación que han mostrado su contradicción: Estado subsidiario versus Estado solidario, los cuales tras un largo proceso político seguido en el período de retorno a la democracia, que se buscó ex profeso extremar sus semejanzas y minimizar las diferencias entre ambas perspectivas, los resultados no muestran avances, ni con mayor incremento de recursos financieros ni con reforma del currículo. El camino a seguir - manteniendo este proceder - parece cada vez más estrecho y difuso, e incluso próximo a agotarse, requiriendo para la búsqueda de solución, analizar nuevos diseños políticos para el sistema educacional chileno, al amparo posiblemente de la discusión de un nuevo rol del Estado.

El período de tiempo en análisis implicó sustentar la provisión de una educación de calidad, inclusiva de la equidad, que generara condiciones ciertas en la población, habilidades, competencias, capacidades y destrezas que les facultaran para reducir en términos significativos las brechas intergeneracionales y la exclusión social, aspectos puestos en entredicho al tenor de los problemas y conflictos que muestra la sociedad chilena en esta materia.

En función de lo expuesto, la relevancia de la experienciachilenaparalaregiónlatinoamericana reside en las lecciones que puedan derivarse de un caso que ha manifestado por casi tres décadas, la búsqueda de una fuerte coherencia entre las políticas económicas de mercado y las políticas sociales que han buscado - casi en los últimos veinte años - reducir sus fallos, impulsado a las políticas educacionales a desempeñar un rol instrumental clave en este aspecto. Esta misión no es menor si se entiende como lo señala NEF (1999/2000) que los sucesos de los años 70 y 80 son partícipes de la contrarrevolución neoliberal, fenómeno que no fue propio solamente del ámbito chileno.

La generación de una Política de Estado en Educación es consistente con el diagnóstico precedente. Sin embargo, esa propuesta implica un Estado políticamente activo como regulador y organizador del sistema educacional en todo su sentido, y que detente las atribuciones para cumplir su tarea. Ello es un contrasentido en un

\footnotetext{
${ }^{7}$ Se entiende crisis como la presencia de dificultades severas que comprometen estratégicamente la existencia del objeto en análisis en los términos actuales cuyo desenlace puede significar su estancamiento como también su progreso.
} 
esquema de Mercado o un modelo de Estado Subsidiario, en consecuencia en alguna medida se exige algo que está fuera del racional que no se quiere o puede cambiar.

El Estado ha manifestado un compromiso medido en los hechos con una educación de calidad. La sociedad en forma mayoritaria reclama para el sector educación "otro Estado". Si embargo en la demanda de una educación de calidad no está del todo asentada en la sociedad civil, en términos de un canal unívoco de expresión y acción al respecto. Tampoco está plenamente enraizada en los gremios docentes -donde se mezcla con reivindicaciones salariales - ni en los de estudiantes y padres, que carecen de la competencia para traducirles de demandas sentidas a requerimientos técnicos. Ello es funcional a una visión tecnocrática de la política, sin embargo, la esperanza de mejoría se confronta con una visión de mercado que no puede destruirse a si mismo para solucionar sus graves problemas. La posibilidad de generar consensos es compleja, la educación es un campo de fuertes intereses ideológicos, también de clase y por ende de poder.

El Estado chileno tiene una baja cuota de poder en este escenario. Su legitimación está cada vez puesta más en duda en términos de ofrecer respuesta eficiente a las necesidades y garantizar a los ciudadanos un sistema educativo de calidad: algo que en la actualidad es ampliamente demandado por la sociedad e insatisfactoriamente cumplido en la educación pública, fenómeno que en el formato de mercado parece irresoluble, salvo que opte - al menos inicialmente - por su auto destrucción.

Bibliografia

AEDO, C. y LARRAÑAGA, O. Educación privada versus educación pública en Chile: calidad y sesgo de selección. Programa de Postgrado en Economía ILADES/Georgetown University, Santiago de Chile, 1995.

AEDO, C. Diferencias sobre escuelas y rendimiento estudiantil en Chile. En: La organización marca la diferencia: Educación y salud en América Latina. Programa de Postgrado en Economía ILADES/ Georgetown University, Santiago de Chile, 1998.

ALMONACID, C., LUZÓN, A. y M. TORRES, M. Cuasi mercado educacional en Chile: el discurso de los tomadores de decisión. Archivos Analíticos de Políticas Educativas, 16 (8). Recuperado [mayo de 2008] de http://epaa.asu.edu/epaa/v16n8/

BRUNNER, J.J. y G. ELACQUA. Capital Humano en Chile. Universidad Adolfo Ibáñez, Santiago, Chile, 2003. CONSEJO ASESOR PRESIDENCIAL PARA LA CALIDAD DE LA EDUCACIÓN. Informe Final. Diciembre de 2006, Santiago de Chile. www.presidencia.cl (consultado diciembre de 2006).

DIPRES. Presupuesto del Ministerio de Educación. Ministerio de Hacienda, Dirección de

Presupuesto, Santiago de Chile, 2003.

DIPPRES. Ejecución Presupuestaria Ministerio de Educación. Ministerio de Hacienda, Dirección de Presupuesto, Santiago de Chile, 2004.

DIRECTIVAS PRESIDENCIALES PARA LA EDUCACIÓN. Presidencia de la República, Chile, 1980.

DONOSO, S.,y. HAWES, G. Eficiencia escolar y diferencias socioeconómicas: a propósito de los resultados de las pruebas de medición de la Calidad de la Educación en Chile. Educação e Pesquisa v. 28, № 2. Pp. 25-40, 2002.

DONOSO, s. y SCHMAL, R. Introducción a la Economía de la Educación. Nociones Fundamentales. Universidad de Talca, Talca, 2008

GALLEGO, F. Competencia y resultados educativos: Teoría y evidencia para Chile. En: Cuadernos de Economía, № 118, pp. 309 -52, 2002.

GONZÁLEZ, P. Estructura Institucional, recursos y gestión en el sistema escolar chileno. En: COX, C. (Editor). Políticas Educacionales en el Cambio de Siglo. La reforma del sistema escolar en Chile. 
Editorial Universitaria, Santiago. Op. cit. Pp. 597 - 660, 2003.

$\mathrm{HSEIH}, \mathrm{CH}, \mathrm{Y}$ M. URQUIOLA. When school competes, how they compete? An assessment of Chile's nation wide school voucher program. World Bank's development group, Washington, 2002.

HOXBY, C. Ideal Voucher. University of Chicago Press, 2002.

JOFRÉ, G. Subvenciones en Educación. Estudios Públicos, Centro de Estudios Públicos, Santiago,

Chile. № 32, Pp. $31-55,1988$.

MARCEL, M, y TOKMAN, C. ¿Cómo se financia la Educación en Chile? Gobierno de Chile, Ministerio de Hacienda, Dirección de Presupuesto. Serie Estudios de Finanzas Públicas, Santiago de Chile, 2005. MIDEPLAN-MINEDUC. Educación y Pobreza. Resultados de la encuesta Casen 2003. www.mideplan. cl/casen (consultada, octubre 27 de 2004).

MINEDUC. Anuario Estadístico 2005. Ministerio de Educación, Santiago de Chile, 2006.

MINEDUC. Anuario Estadístico 2006. Ministerio de Educación, Santiago Chile, 2007.

MINEDUC. Resultados de las Pruebas simce 2007. Ministerio de Educación, Santiago de Chile, 2008. MIZALA, A. y ROMAGUERA, P. Regulación, incentivos y remuneraciones de los profesores en Chile. En: COX, C. (Ed) Políticas Educacionales en el Cambio. Op. Cit. Pp. 519 -568, 2003.

MIZALA, A. y ROMAGUERA, P. El Sistema Nacional de Evaluación del Desempeño docente (SNED) en Chile. En: BID - PREAL. Maestros en América Latina: nuevas perspectivas sobre su formación y desempeño. Santiago, Chile, Pp.389-407, 2004.

NEF, J. El Concepto de Estado Subsidiario y la Educación como Bien de Mercado: Un Bosquejo de Análisis Político. Revista Enfoques Educacionales. Vol.2 Nº, Departamento de Educación, Facultad de Ciencias Sociales, Universidad de Chile, 1999-2000.

OCDE. Chile. Revisión de las políticas Nacionales de Educación. Organización para la Cooperación y el Desarrollo. París y Ministerio de Educación, Chile, 2004.

PNUD. Desarrollo Humano en Chile 2002. Programa de las Naciones Unidas, 2003.

RAWLS, J. Justicia como Equidad. Editorial Universitaria Tecnos, Madrid, 1986.

ROJAS, P. Renumeraciones de los Profesores en Chile. Estudios Públicos N ${ }^{\circ} 71$, Centro de estudios Públicos, Santiago, Chile, 1998. Pp.122-175.

SAPELLI, C. Introducción: La Economía de la Educación y el Sistema Educativo Chileno.

Cuadernos de Economía, № 118, 2002, Pp. 281 -296.

SAPELLI, C. y VIAL, B. The performance of private and public schools in the Chilean voucher system. Cuadernos de Economía, 2002, № 118, pp. 423 -52.

TOKMAN, A. Is private education better? Evidence from Chile. Banco Central de Chile, 2001. 